\title{
El Sistema à la croisée des chemins
}

Principes et perspectives d'un réseau mondial

El Sistema at the crossroads. Principles and perspectives of a global network

El sistema en la encrucijada. Principios y perspectivas de una red mundial

\section{Maria Majno}

\section{OpenEdition}

\section{Journals}

Édition électronique

URL : https://journals.openedition.org/ries/5986

DOI : 10.4000/ries.5986

ISSN : 2261-4265

\section{Éditeur}

France Education international

\section{Édition imprimée}

Date de publication : 1 septembre 2017

Pagination : $139-148$

ISBN : 978-2-85420-615-9

ISSN : $1254-4590$

Référence électronique

Maria Majno, «El Sistema à la croisée des chemins », Revue internationale d'éducation de Sèvres [En ligne], 75 | septembre 2017, mis en ligne le 01 septembre 2019, consulté le 25 juin 2021. URL : http:// journals.openedition.org/ries/5986; DOI : https://doi.org/10.4000/ries.5986 


\title{
El Sistema à la croisée des chemins
}

\section{Principes et perspectives d'un réseau mondial}

\author{
Maria Majno* \\ Association SONG onlus Sistema in Lombardia, \\ Sistema Europe
}

\author{
Und in dem "Wie»... \\ Da liegt der ganze Unterschied ${ }^{1}$ \\ H. von Hoffmansthal \\ Der Rosenkavalier, Acte 1
}

Travailler sur « El Sistema » impose de distinguer la légende et l'histoire, l'action et son retentissement, l'extension et la persistance, l'engouement et la réalité, la passion et la militance, les souhaits et les résultats objectifs. La naissance du projet, avec 11 musiciens, dans un garage de Caracas, la multiplication des participants dès le lendemain, la propagation quasi-miraculeuse des années suivantes, les centaines de "núcleos $»^{2}$ sur tout le territoire vénézuélien, y compris dans les zones les plus éloignées, puis les tournées des principaux orchestres du Venezuela dans des salles célèbres et les soirées qui se terminent immanquablement par une explosion de joie, telles sont les manifestations d'une mythologie attrayante et longtemps alimentée, il faut en convenir, par une propagande évidente.

Le projet a connu son apogée au moment de la désignation, en 2007, de Gustavo Dudamel, son plus célèbre talent, à la direction musicale du Los Angeles Philharmonic, puis des « résidences » en 2013 au Festival de Salzbourg et à la Scala de Milan pour EXPO 2015. Ces deux cycles ont célébré de façon concluante toutes les facettes de la production officielle d'El Sistema, en présentant les différentes générations orchestrales, des vétérans du "Simón Bolívar» à l'Orchestre "Infantil » (âge maximal 13 ans). ${ }^{3}$

Aujourd'hui le Venezuela connaît une situation dramatique avec $80 \%$ de la population (août 2017) vivant au-dessous du seuil de pauvreté, privée de nourriture et de soins.

\footnotetext{
* Maria Majno exprime ses plus vifs remerciements à Chara lacovidou, Isabelle Joris et Michel Starobinski pour leurs précieuses observations.

1. "Et dans le "comment" se trouve toute la différence ".

2. Les structures fonctionnelles, éducatives, artistiques et administratives d’El Sistema sont appelées « noyau ».

3. L'enregistrement de la Première Symphonie de Mahler par l'Orquesta Infantil sous la direction de Sir Simon Rattle (Unitel, 2013) compte parmi les documents les plus convaincants d'une qualité interprétative qui ne craint guère les comparaisons.
} 
Auparavant, les résultats d'El Sistema ont été indéniables : El Sistema a mobilisé au Venezuela jusqu'à 700000 jeunes par an, et trois fois plus si l'on prend en compte la totalité de son histoire. Certes, tous ne sont pas devenus musiciens mais il est évident qu'une telle masse a dû laisser des traces positives. C'est donc plutôt sur le plan international qu'il faut chercher à situer aujourd'hui l'héritage de José Antonio Abreu. Le fondateur d'El Sistema a incontestablement sa place parmi les personnages ayant le plus contribué aux fondements, à la pratique, à la réflexion sur l'éducation musicale de notre époque.

\section{L'ACTUALISATION DES PRINCIPES FONDATEURS}

Le fait que la pratique musicale d'ensemble est le meilleur foyer de démocratie et de progrès est le premier présupposé de El Sistema.

Par essence, l'orchestre et le chœur sont bien plus que des structures artistiques. Ce sont des exemples et des écoles de vie sociale, car chanter et jouer ensemble comporte une coexistence profonde qui tend vers la perfection et l'excellence [...]. (Abreu, 2009)

Les variations sur ce motif abondent dans les propos du créateur d'El Sistema. Jusqu'à ses dernières interventions devant le grand public en 2013, 140 l'humaniste Abreu a puisé dans la philosophie classique, comme les fameux énoncés d'Aristote et de Platon sur l'importance de la musique dans l'éducation. La formation à la beauté, dont on devient affamé ou assoiffé dès qu'on en a goûté la richesse immatérielle, affranchit de la vraie pauvreté, celle « de ne se sentir personne ». Ainsi, El Sistema a été conçu comme

un programme de récupération sociale et transformation culturelle profonde [...] adressé sans distinctions, mais en priorité aux groupes vulnérables et en danger ${ }^{4}{ }^{4}$ (Abreu, 2009)

Abreu croit en la compensation des inégalités par l'accès à la pratique, par les opportunités de succès, au progrès comme agent d'affranchissement, à l'engagement comme vecteur de discipline et de respect.

L'effet d'El Sistema se fait ressentir dans trois domaines fondamentaux : dans la sphère personnelle et sociale, dans la famille et dans la communauté [...] La musique devient une source pour développer les dimensions de l'être humain [...] ; les avantages émotionnels et intellectuels sont énormes.

À partir du discours d'acceptation de J. A. Abreu au prix TED, qui reste une référence pour sa pensée, plusieurs auteurs (dont les «Abreu Fellows»), se sont efforcés de lister les valeurs fondamentales impliquées dans El Sistema.

4. Voir : www.fundamusical.org.ve/ 
El Sistema n'est ni un programme, ni un curriculum, ni une pédagogie. C'est une quête. [...] Plusieurs programmes valables d'apprentissage musical partagent des valeurs et des pratiques semblables. Or, 42 années d'exploration pour cerner les façons les plus efficaces de développer la jeunesse à travers ces priorités ont produit des résultats sans précédents. [...] En s'engageant dans ce travail, on aspire à tous ces objectifs [...] et on trouve son propre chemin : les idéaux sont globalement partagés, adaptés et incarnés dans chaque culture spécifique. (Booth, 2017).

La «systématisation » proposée par Eric Booth suit une série de dix principes, sorte de décalogue que nous proposons de confirmer ou compléter avec les acquis de notre expérience, ayant été un acteur principal de l'établissement d'El Sistema en Lombardie en Italie et de la création du réseau européen ${ }^{5}$. Tout au long de la diffusion du modèle vénézuélien aux autres pays et continents, nous avons pu bénéficier des leçons directes ou indirectes tirées de l'adaptation d'El Sistema aux divers territoires.

\section{Une mission de changement social}

Tocar y Luchar («jouer et lutter») : les deux verbes de cette devise éclairent différemment les objectifs culturels et sociaux d'El Sistema, selon les contextes respectifs. Si El Sistema est né au Venezuela comme une contreproposition à la dégradation et à la tentation de la violence, sa dimension de lutte et de résistance se traduit en « jeu », dans d'autres langues, sans pour cela renoncer à la dimension d'engagement. Ainsi, la pratique instrumentale évoquée par «tocar " rappelle tangiblement le pouvoir émotionnel direct de la musique, sa capacité à «toucher » immédiatement, au point que l'Orquestra Geraçaõ Sistema Portugal en a tiré sa devise : "Aprendendo música, para tocar vídas».

\section{Accès et excellence : dépasser la dichotomie}

Les conditions premières dans le projet d'origine sont l'absence de sélection et la gratuité : impliquer le plus grand nombre possible d'enfants sans demander de contribution financière et en même temps viser le sommet, avec l'ambition de l'excellence. Le contraste entre ces finalités a longtemps dominé le débat, jusqu'à risquer de polariser les approches entre le but «musical » et le but « social ». La radicalisation d'un tel raisonnement peut conduire au refus des " niveaux» qui jouent une part essentielle dans l'idée de croissance implicite dans l'éducation. Maintes initiatives inspirées par El Sistema ont résolu la quadrature du cercle en recourant à un critère de qualité également apte à assurer la motivation des participants: pas de progrès sans persévérance, pas de 
persévérance sans la satisfaction d'augmenter ses possibilités et la richesse des résultats. L'image de la pyramide convient aussi bien au modèle qu'à ses dérivations : les deux mots clé s'imbriquent facilement dans le but d'une construction à la fois solide et élevée ${ }^{6}$.

\section{L’environnement du núcleo comme ambiance et contexte}

Les centres didactiques d'El Sistema sont nés comme une gigantesque "permanence " après-scolaire proposant une alternative aux risques de la rue dans les Barrios : violence, crime, danger de mort. Cette coexistence physique crée un havre de sécurité, fermé à la force mais ouvert à la communauté. Dans d'autres pays, moins affectés par cette absence d'opportunités, l'image du noyau représente bien l'échange entre le centre et les alentours, les protagonistes de l'apprentissage et ceux qui les soutiennent, dans une atmosphère de solidarité productive et engagée. Même les écoles de musique préexistantes ont voulu se transformer. Les méthodes traditionnelles se sont hybridées avec les innovations de l'enseignement collectif, élargissant ainsi les horizons pédagogiques avec des adaptations propres au milieu égalitaire. Ce n'est pas un hasard si le plus authentique Nucleo en Lombardie s'est développé dans un centre social nommé «Barrios».

\section{Intensité, fréquence des performances publiques : la contagion de la collectivité}

L'intensité et la fréquence de la pratique dans les núcleos au Venezuela ont attiré autant - sinon plus - de critiques que d'approbation. Quatre heures par jour, six jours par semaine, sans compter les séminaires et les répétitions exceptionnelles offrent une cible évidente pour la critique, qui voit dans cette ténacité un jeu de pouvoir dissimulé. Toutefois, cette cadence soutenue s'avère indispensable pour assurer de le progrès à démontrer en public (voir point 8).

\section{Utilisation de l'ensemble : le paradoxe des talents intégrés}

Simultanément, l'enseignement de groupe permet une éducation à la vie collective avec ses règles, et offre une ressource moins coûteuse pour toucher le plus grand nombre. Ainsi, selon le principe du "peer teaching \& learning", l'enseignement et l'apprentissage par les pairs, toute compétence apprise est aussi

6. L'éventail des «Sept Dimensions » élaboré pour la pédagogie commune propédeutique aux projets du SEYO Sistema Europe Youth Orchestra (Drive \& Dance, Flow, Love in Sound...) a été conçu délibérément pour être appliqué aussi bien aux instrumentistes juniors qu'aux plus avancés (www.sistemaeurope.org/seyo). 
apte à être enseignée, et celui qui sait jouer quatre notes sera encouragé à les transmettre à celui qui en sait deux. Des enquêtes récentes se sont donné pour but de mesurer les bénéfices de la musique d'ensemble en fonction du style de répétition, des sources de perception multiple, de la tolérance à la réitération, de l'attention distribuée, de l'accélération de l'empathie (Brice Heath, 2016). Il ne faut toutefois pas oublier les besoins de l'individu, surtout s'il montre du talent. L'un des problèmes les plus fréquemment discutés est précisément la compatibilité avec l'enseignement individuel, indispensable tôt ou tard pour les talents les plus manifestes. Une solution consiste alors à faire de l'attention particulière une récompense non exclusive mais valorisante.

\section{Le modèle CATS :}

\section{Citizen Artist Teacher Scholar}

L'un des résultats des premiers «Abreu Fellows» est l'élaboration du quadrilatère qui définit le rôle de ceux qui guident la formation dans le contexte d'El Sistema : l'enseignant musicien agit en même temps dans le sillage des teaching artists, qui trouvent dans leur vocation éducative une motivation ultérieure à leur engagement esthétique et social (Booth, 2009). À ces acteurs éclectiques vient s'ajouter une troisième dimension, qui comporte des visées ultérieures de recherche (scholars). Enfin, des passerelles se construisent en direction des citizen artists (artistes citoyens), dont on observe l'essor et qui font écho concrètement à une phrase d'Abreu souvent citée comme point de départ d'El Sistema :

Nous ne cherchons pas à former de bons musiciens mais de meilleurs citoyens.

\section{Une mise à jour continue de la pédagogie}

L'éducation et l'enseignement partagent la caractéristique de ne pas avoir de fin : s'il existe dans El Sistema une " méthode " (terme à prendre avec circonspection lorsqu'il s'agit d'une stratégie), c'est bien la gradation séquentielle de l'itinéraire pédagogique, et la conscience que le processus ne s'arrête pas avec l'achèvement de la maîtrise instrumentale ${ }^{7}$. Les spécificités de l'approche collective requièrent en premier lieu une formation particulière : même les mentors qui abordent ce mode didactique avec un abondant bagage technique admettent qu'il n'est guère évident de "savoir s'y prendre ». Quelle que soit la portée du réseau, le besoin le plus constamment évoqué par les acteurs est celui d'un accompagnement plus dense, subtil et expérimenté : une étude de Sistema Europe a confirmé que ce souhait reste au premier plan jusque dans la diversité même des projets.

7. Les enseignants des différents projets Sistema tendent à leur tour à adapter les méthodes de didactique musicale à leurs propres styles et environnements, le plus souvent en s'inspirant des approches de Dalcroze, Kodály, Orff et Suzuki. 


\section{L'implication de la famille et de la collectivité}

Parmi les ingrédients de la réussite du Sistema, l'appui de la famille - et celui de l'école - est fondamental dans toutes ses phases : l'impulsion au démarrage, le dépassement des obstacles, la stabilisation de l'effort, la dynamique de l'ambition. C'est le public qui apprécie et récompense, dans un cercle de motivation réciproque. Mais la proximité des familles est aussi un outil puissant pour rapprocher et construire le public de demain : les préjugés contre la musique «savante " se voient bien mieux combattus par cette complicité instinctive que par des interventions exogènes. La discipline quotidienne est allégée par le corollaire de performances publiques fréquentes. Celles-ci ne sont pas perçues comme des épreuves redoutables mais plutôt comme des occasions de mettre en valeur les acquis, donnant lieu à une sorte de contagion réciproque entre exécutants et nouveaux auditeurs, conquis par la découverte expressive d'émotions révélées.

\section{Liens et réseaux pour mobiliser la solidarité}

L'adaptation au territoire conditionne directement la durabilité de chaque núcleo. Une évaluation préliminaire de la volonté d'une adoption permanente par la communauté, au-delà des enthousiasmes salutaires mais transitoires, est une étape indispensable pour les promoteurs d'El Sistema, avant d'implanter un nouveau projet. C'est l'intégration sur le territoire qui assure une motivation organique à prendre soin sur le long terme d'un corps d'abord étranger, en l'assistant lors de transitions difficiles ou devant des obstacles à sa soutenabilité et à sa permanence dans le temps. Périodiquement, la question se pose : quand les ressources tarissent, est-ce envisageable de s'adresser aux enfants et aux familles pour leur dire que tout cela était réussi, mais qu'hélas c'est maintenant fini ? C'est là qu'entre en jeu la mobilisation des ressources complémentaires. Tout centre didactique est un acteur de la décentralisation, aussi bien qu'un membre des relations globales, ce qui assure aussi un transfert adéquat d'un núcleo à un autre, la mobilité personnelle étant aussi à respecter. Le travail sur le territoire comporte nécessairement des échanges vers une construction unifiée et cohérente, qui puisse ensuite inspirer également l'individu en dehors de la dimension musicale. Sans oublier que cette approche intégratrice permet de surmonter les tensions pouvant se manifester avec les autres structures didactiques établies, telles que les conservatoires ou les écoles de musique existantes.

\section{Ambition et réussite : un cercle inclusif}

Comme le rappelle souvent Marshall Marcus (2017), fondateur, entre autres, de Sistema Europe, l'ambiance d'El Sistema fait oublier la crainte de l'échec et apprécier l'erreur comme facteur d'expérience. Ainsi le dialogue est 
réellement ouvert aux capacités particulières et à l'inclusion du handicap physique ou mental : selon Naybeth García et Jhonny Gómez, fondateurs du Programa de Educación Especial, "l'âme ne connaît pas d'incapacité ». En parallèle aux justes efforts de la validation scientifique, cela nous rappelle que l'art conserve un fond irréductible et non mesurable, constitutif de l'unicité de la culture humaine. C'est la dimension esthétique qui est capable à son tour de provoquer le changement social.

\section{Une stratégie en mouvement}

Ce catalogue n'est évidemment pas la seule façon d'énumérer et de commenter les caractéristiques d'El Sistema. On pourrait aussi se concentrer sur une rose des vents dont les points cardinaux sont l'intégration sociale, la sensibilisation culturelle, l'éducation musicale et la qualité artistique (figure 1), ou produire un kaléidoscope énergique et joyeux de 21 traits constitutifs, comme l'a proposé Marshall Marcus (2017). Ce qui nous semble constituer l'essence de tous ces propos est la considération qu'il s'agit d'axes dynamiques plutôt que de points fixes, et qu'El Sistema est une stratégie en mouvement.

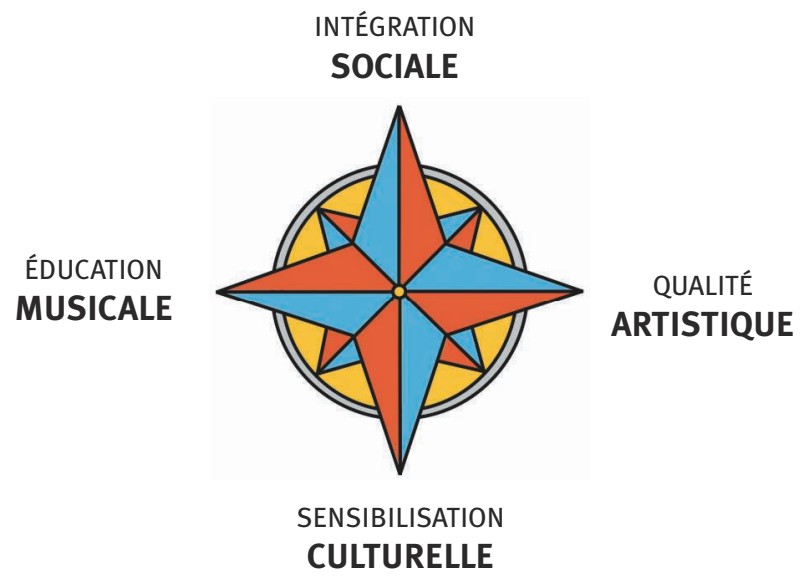

\section{DE LA CRITIQUE À LA PERSPECTIVE}

Cela faisait presque quarante ans qu'on disait du bien d'El Sistema, et il était certainement temps d'engager une discussion objective sur l'ensemble de ses démarches et de ses résultats. Ceci à plus forte raison considérant son expansion à cinq continents (la Russie semble être le seul grand État sans projet inspiré d'El Sistema). Cette tâche critique a été assumée par Geoff Baker, enseignant- 
chercheur spécialisé dans les musiques latino-américaines. Son ouvrage El Sistema - Orchestrating Venezuela's Youth (2014) expose le parcours entre l'enthousiasme initial et la découverte d'abîmes négatifs lors son enquête au Venezuela. ${ }^{8}$ Ses critiques ont eu un grand retentissement, mais les défenseurs d'El Sistema ont souligné de leur coté, les limites d'une recherche « ethnographique » qui procède sur la base d'une imbrication étroite entre le chercheur et le terrain concerné (Kenyon, 2015). La querelle se poursuit mais les arguments invoqués s'usent au fur et à mesure que le temps passe ${ }^{9}$ et, en 2017, Baker a déclaré sur son blog: " je ne pense pas, une seule seconde, qu'El Sistema manque d'aspects positifs ».

Plus que jamais, le dialogue doit donc rester concret, ouvert à une révision constante, voire radicale, mais constructive. Il faut admettre que tout pouvoir comporte un risque d'instrumentalisation et, dans ce contexte, on a vu émerger des formulations centrées sur les "droits ", comme les travaux du Salzburg Global Seminar n. 479 (2011), qui ont donné lieu à la déclaration "The Value of Music: The Right to Play."

C'est l'élan pour contribuer à l'établissement de ce droit, bien plus qu'à une dynamique de pouvoir, qui se révèle capable d'éveiller la motivation solidaire des orchestres et des salles de concerts, et des artistes sensibilisés. Ces alliés sont fondamentaux aussi bien pour la diffusion que pour la qualité du projet El Sistema, et un panorama de leurs contributions devrait faire l'objet d'une étude ultérieure ; nous en donnons des exemples en fin d'article. ${ }^{10}$

Une autre alliance prometteuse est celle des scientifiques. Nous nous bornerons ici à évoquer la série de congrès "Neuromusic", et le bulletin électronique bimensuel Neuromusic News, gérés par la Fondazione Mariani pour la neurologie pédiatrique. Lors du dernier congrès, à Boston sur le thème "Music, Sound and Health», une session entière a été consacrée aux bénéfices extramusicaux de l'apprentissage musical, dont trois exposés ont touché de près le contexte d'El Sistema.

Le milieu scolaire et parascolaire offre la pépinière la plus adaptée pour ce genre d'études. Dans le cadre du Sistema en Lombardie, The San Siro Project: musical training, cognitive and emotional development (Danelli, Paulesu et al., en cours d'élaboration) examine justement l'évolution des adolescents entre 11 et 14 ans dans une étude longitudinale concernant les axes cognitivo-émotionnel, comportemental et socio-culturel.

8. Rappelons que le point de départ de Baker est une contestation de fond de la structure de l'orchestre comme modèle de collaboration et de démocratie : il y voit au contraire un chaudron pour des jeux de pouvoir malsains. Ce présupposé est en effet difficilement réconciliable avec la conception valorisante des ensembles musicaux comme foyers d'écoute, de respect réciproque et d'intentions synergiques.

9. Voir le site de Jonathan Govias, par exemple.

10. La détermination de Claudio Abbado constitue probablement l'exemple le plus éloquent de force de rassemblement à cet égard, puisqu'il a soutenu l'introduction et le développement d'un Sistema national pour l'Italie (Abbado, 2012), mais aussi pour l'Europe, compte tenu de son engagement pour les orchestres de jeunes Mahler Jugendorchester et European Union Youth Orchestra. 
Par ailleurs, un recensement des contributions académiques est à l'ordre du jour, dans l'effort de suivre les travaux sur les ramifications multiples du Sistema, alors que ces travaux augmentent à un rythme tel qu'il se révèle quasiment impossible de se maintenir à jour. Mais les tentatives même incomplètes aboutissent de toute façon à des résultats stimulants (voir les principales platesformes en fin d'article) ${ }^{11}$.

En passant des critiques constructives aux orientations de recherche et aux perspectives plus générales, on peut aussi lire la situation d'origine et son rayonnement ultérieur comme une "crise de croissance » de grande amplitude. À plus forte raison parce que les núclei (ou les structures analogues), une fois établis, non seulement demandent à être soutenus, mais entraînent à leur tour une augmentation de la demande. La gestion présuppose donc une sorte d'ingéniosité inlassable, pour multiplier les ressources sans en disposer nécessairement a priori. ${ }^{12}$ Les managers des projets se réclament souvent d'un concept imaginatif de "valeur ajoutée ", selon lequel la croissance personnelle et la satisfaction évoquées par les professionnels participants offrent déjà en elles-mêmes des éléments importants de récompense.

Par contraste, l'intention de José Antonio Abreu, qui a été économiste autant que musicien et ministre de la culture, est que l'investissement dans El Sistema comporte aussi des bénéfices économiques, notamment pour la prévention (abandon scolaire, intimidation, criminalité). On attend toujours l'élaboration d'une recherche apparemment en cours sur les indicateurs, confiée à la collaboration entre El Sistema Venezuela et la Banque mondiale. Il reste encore beaucoup de travail à faire sur la démonstration et la publication des résultats.

\section{BIBLIOGRAPHIE}

ABBADO C. (2012) : Préface à A. RADAELLI, La musica salva la vita, Milan: Feltrinelli.

ABREU J.A. (2009) : Discours pour la réception du Prix TED. [https://goo.g/BGsyas]; [https://goo.gl/i34w2q]

ABREU J.A. (2013) : Discours d'inauguration du Festival de Salzbourg. [https://www.youtube.com/watch?v=VTjfx2mp-XI]

BAKER G. (2014) : El Sistema: Orchestrating Venezuela's Youth, Oxford, UK: Oxford BOOTH E. (2009, 2013, 2017) : [http://ericbooth.net/category/el-sistema]

BRICE HEATH S. (2016) : "The Benefits of Ensemble Music Experience (and why These Benefits Matter so Much in Underserved Communities) " in El Sistema-Music for Social Change, C. Witkowski (ed.), London, New York: Omnibus Press, p. 73-88.

11. Parmi les articles récents et prometteurs, quoique provisoires, nous citons celui du partenariat Longy School Bard College / WolfBrown, relié au réseau de Sistema USA. [https://goo.gl/LZF3q1/] ; [https://goo.gl/Sjid1x/].

12. Il est impressionnant de lire, dans le dossier de presse sur la « Nouvelle ambition de déploiement national 20162019 » du projet Démos de la Philharmonie de Paris, que le coût annuel pour un orchestre est estimé à 260000 Euros. Ce montant correspond, dans d'autres environnements, au budget annuel d'un réseau national tout entier. 
KENYON N. (2015) : "The Triumph of a Musical Adventure », The New York Review of Books, 24 septembre 2015, p. 74-76.

MARCUS M. (2017) : «Sistema Europe: Origins, Approaches and Diversity (powerpoint) : [https://marshallmarcus.wordpress.com/PDP2017]

SALZBURG GLOBAL SEMINAR (2011) : The Value of Music: The Right to Play, [https://g0o.g/Pta7fG]

TUNSTALL T. \& BOOTH E. (2016) : Playing for Their Lives - The Global El Sistema Movement for Social Change Through Music, New York, NY : W.W. Norton.

\section{SOURCES D'INFORMATION COMPLÉMENTAIRES}

\section{Artistes et organisations}

Fondation Katia et Marielle Labèque : [http://www.fondazionekml.org]

Göteborg Symphony Side By Side: [https://www.gso.se/en/barn-unga/side-by-side]

Los Angeles Phiharmonic, YOLA at HOLA: [https://www.laphil.com/education/yola/hola]

Philharmonie de Paris, Démos : [http://demos.philharmoniedeparis.fr]

\section{Congrès et conférences}

Take a Stand (Los Angeles Philharmonic) : [https://goo.g/TGhRXS]

Pratiques collectives en orchestre et accès à la culture (Démos, Philharmonie de Paris) : [https://goo.gl/qc5mi4]

\section{Lettres d'information et blogs}

The Ensemble : [theensemblenewsletters.com]

Neuromusic News (Fondazione Mariani) : [https://goo.glXjnGah]

Playing for Their Lives : [http://playingfortheirlives.com]

\section{Recherche}

Porticus - CAL Community Arts Lab : [https://www.porticus.com/en/home]

SERA - Sistema Evaluation and Research Archive : [https://goo.glfc9Arv]

SIMM Social Impact of Making Music (Guildhall School Music) : [https://goo.gl/Bkon7r]

Sistema Global Review I \& II : [http://sistemaglobal.org/literature-review]

\section{Réseaux et ressources}

Sistema Connect : [http://www.sistemaconnect.org]

Sistema Europe : [www.sistemaeurope.org]

Sistema Global : [www.sistemaglobal.org] ; [http://sistemaglobal.org/resources]

Sistema USA : [https://www.elsistemausa.org] 